\title{
Spin-photo-currents generated by femtosecond laser pulses in a ferrimagnetic GdFeCo/Pt bilayer
}

Cite as: Appl. Phys. Lett. 110, 072402 (2017); https://doi.org/10.1063/1.4976202

Submitted: 13 December 2016 . Accepted: 30 January 2017 . Published Online: 13 February 2017

T. J. Huisman, C. Ciccarelli, A. Tsukamoto, R. V. Mikhaylovskiy, Th. Rasing, and A. V. Kimel
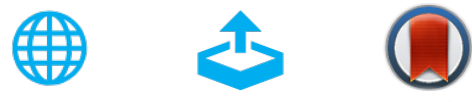

View Online

\section{ARTICLES YOU MAY BE INTERESTED IN}

Perspective: Ultrafast magnetism and THz spintronics

Journal of Applied Physics 120, 140901 (2016); https://doi.org/10.1063/1.4958846

Ultrabroadband single-cycle terahertz pulses with peak fields of $300 \mathrm{kV} \mathrm{cm}^{-1}$ from a metallic spintronic emitter

Applied Physics Letters 110, 252402 (2017); https://doi.org/10.1063/1.4986755

Conversion of spin current into charge current at room temperature: Inverse spin-Hall effect

Applied Physics Letters 88, 182509 (2006); https://doi.org/10.1063/1.2199473

\section{Applied Physics Letters}

Mid-IR and $\mathrm{THz}$ frequency combs special collection

Read Now! 


\title{
Spin-photo-currents generated by femtosecond laser pulses in a ferrimagnetic $\mathrm{GdFeCo} / \mathrm{Pt}$ bilayer
}

\author{
T. J. Huisman, ${ }^{1}$ C. Ciccarelli, ${ }^{2}$ A. Tsukamoto, ${ }^{3}$ R. V. Mikhaylovskiy, ${ }^{1}$ Th. Rasing, ${ }^{1}$ \\ and A. V. Kimel ${ }^{1}$ \\ ${ }^{1}$ Institute for Molecules and Materials, Radboud University Nijmegen, 6525 AJ Nijmegen, The Netherlands \\ ${ }^{2}$ Cavendish Laboratory, University of Cambridge, CB3 OHE Cambridge, United Kingdom \\ ${ }^{3}$ College of Science and Technology, Nihon University, 7-24-1 Funabashi, Chiba, Japan
}

(Received 13 December 2016; accepted 30 January 2017; published online 13 February 2017)

Using THz emission spectroscopy, we detect spin-photo-currents from a ferrimagnetic amorphous alloy $\mathrm{GdFeCo}$ to an adjacent Pt capping layer. The currents are generated upon excitation of a $\mathrm{GdFeCo} / \mathrm{Pt}$ heterostructure with femtosecond laser pulses. It is found that the polarization of the spin-polarized current is determined by magnetic sublattice sensitivity rather than the total magnetization, allowing for spin-polarized current generation when the net magnetization is zero. Published by AIP Publishing. [http://dx.doi.org/10.1063/1.4976202]

Spintronics, i.e., the functional use of the coupling of spins and movement of electrons, has developed in the last decades from a scientific discovery to a standard solution in data memory and logic technology. ${ }^{1}$ Very recently, the idea of antiferromagnetic spintronics has been suggested, with attractive features including insensitivity to disturbing magnetic fields, absence of parasitic interactions between neighboring antiferromagnetic elements, and intrinsically faster spin dynamics compared to ferromagnets. ${ }^{2}$ Despite several theoretical predictions, experimental verification of spincurrents from antiferromagnets has so far been very challenging. As a result, the feasibility of an effective generation of spin-currents from a material with no net magnetization is still questionable.

A new approach towards antiferromagnetic spintronics may come from metallic heterostructures, in which a ferromagnetic layer is brought in contact with a metallic nonmagnetic layer. In such structures, a spin-polarized current can be generated upon femtosecond laser excitation. ${ }^{3-6}$ The ultrashort pulse launches an intense diffusion of spins from the magnetic to the non-magnetic layer due to the excitation of hot electrons with spin-dependent velocities. ${ }^{7}$ Here, we study the effect of the net magnetic moment on laserinduced spin-polarized currents. For this, we have chosen the ferrimagnetic metallic alloy GdFeCo. This alloy has two magnetic sublattices ( $\mathrm{Gd}$ and $\mathrm{FeCo}$ ), which are coupled antiferromagnetically. Due to different $\mathrm{Gd}-\mathrm{Gd}$ and $\mathrm{Fe}-\mathrm{Fe}$ exchange constants, the magnetizations of these two sublattices have different temperature dependences. As a result, the net magnetization of the material can be effectively tuned by changing the temperature of the alloy. At the so-called magnetization compensation temperature $\left(\mathrm{T}_{\text {comp }}\right)$, the magnetization of the $\mathrm{FeCo}$ magnetic sublattice exactly balances that of the Gd and the net magnetization becomes zero. For $\mathrm{T}>\mathrm{T}_{\text {comp }}$, the net magnetization is dominated by FeCo, while for $\mathrm{T}<\mathrm{T}_{\text {comp }}$, the main contribution to the net magnetization is given by the magnetic sublattice of $\mathrm{Gd}$. The goal of this work is to reveal how the spin-photocurrent from a GdFeCo alloy changes upon changing the net magnetization.
To achieve this goal, we employed the fact that a femtosecond laser excitation of a $\mathrm{GdFeCo} / \mathrm{Pt}$ heterostructure leads to a spin diffusion from $\mathrm{GdFeCo}$ to $\mathrm{Pt}$ on a picosecond to sub-picosecond timescale, i.e., at $\mathrm{THz}$ frequencies. ${ }^{3-11}$ Due to the strong spin-orbit interaction in Pt, this layer works as a transformer of the pulse of spin-polarized electrons into a charge current pulse in the plane of the structure. The latter is a source of $\mathrm{THz}$ radiation in accordance with the Maxwell equations. By performing $\mathrm{THz}$ emission spectroscopy, one thus acquires the mean for detection of the femtosecond spin-photo-currents from the GdFeCo to the Pt layer.

The sample that we have used in our experiments is shown in Fig. 1(a). It consists of a $20 \mathrm{~nm}$ thick layer of $\mathrm{Gd}_{0.25}\left(\mathrm{Fe}_{0.875} \mathrm{Co}_{0.125}\right)_{0.75}$ grown in contact to a $2 \mathrm{~nm}$ layer of Pt. This multilayer sample is grown on a glass substrate by magnetron sputtering, using a $5 \mathrm{~nm}$ thick SiN layer as seed, and a $5 \mathrm{~nm}$ thick layer of SiN layer as capping to prevent oxidation. The particular composition of our alloy results in a uniaxial anisotropy with the out-of-plane $\hat{z}$ direction being the easy axis. Therefore, when no magnetic field is applied, the magnetic moments of both $\mathrm{FeCo}$ and Gd lay along the out-of-plane easy axis.

The full setup that we used for our experiment is depicted in Fig. 1(a). An amplified laser system produces 50 fs short laser pulses, which are split into two beam paths. One beam path is used to stimulate a spin-photo-current from the alloy. The spin-photo-current in the Pt layer results in the emission of $\mathrm{THz}$ radiation, which is collected and refocused using parabolic mirrors onto a $1 \mathrm{~mm}$ thick $\mathrm{ZnTe}$ crystal. By using electro-optic sampling, ${ }^{12}$ we detected the time-dependence of the electric field of this radiation. The polarization axis of the emitted $\mathrm{THz}$ signal is given by the vector product $\hat{\mathbf{z}} \times \boldsymbol{\sigma}_{i p}$, where $\boldsymbol{\sigma}_{i p}$ is the projection of the injected spin-polarization on the plane of the sample. To gain nonzero $\boldsymbol{\sigma}_{i p}$ in the studied structures, we apply an inplane magnetic field $\mathbf{B}_{\text {ip }}$ that tilts the magnetic moments away from the out-of-plane easy axis.

In Fig. 1(b), we show examples of the measured electric field as a function of time. It is seen that turning the sample around the vertical axis over $180^{\circ}$ results in the reversal of 
(a)

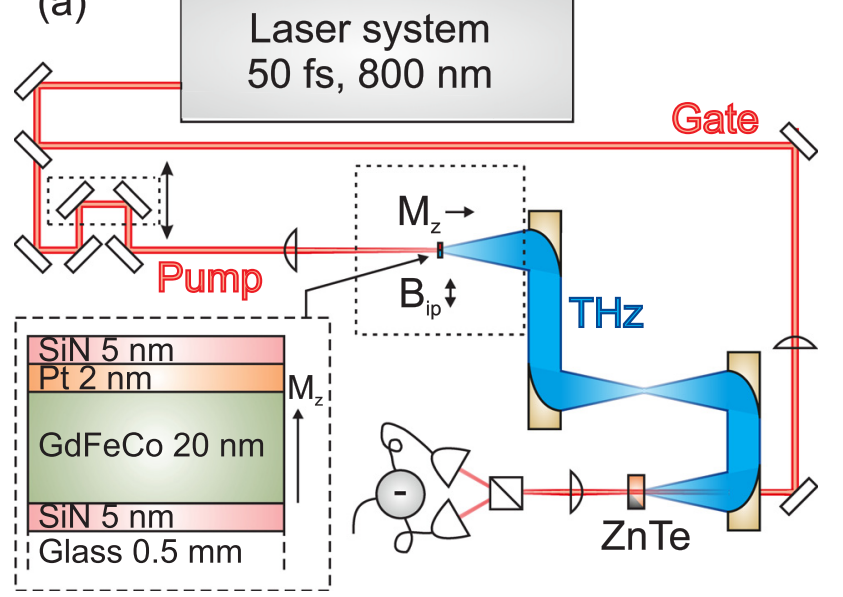

(b)

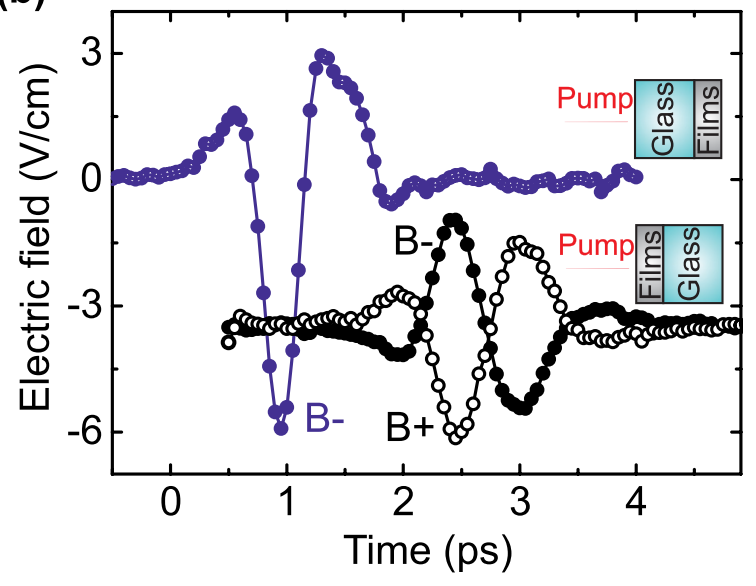

FIG. 1. Schematics of the experiment and room temperature measurements. (a) Intense laser pulses, the pump, are incident on the GdFeCo/Pt sample placed in a $7 \mathrm{~T}$ cryostat. The sample emits THz radiation, which is collected using parabolic mirrors and measured using electro-optic sampling and a polarization balanced detector scheme. The sample structure is shown left at the bottom. (b) Observed traces of the electric field of the emitted THz radiation. The blue line corresponds to the electric field of the emitted THz radiation when the pump is incident from the glass side of the substrate. The black traces (shown with a vertical offset) correspond to the electric field of the emitted $\mathrm{THz}$ radiation when the pump is incident from the side of the metallic layers. $\mathrm{B}-$ and $\mathrm{B}+$ correspond to an in-plane applied field of -0.5 and $0.5 \mathrm{~T}$, respectively.

the sign of the emitted $\mathrm{THz}$ traces. Furthermore, it is seen that the emitted $\mathrm{THz}$ traces also reverse the sign upon reversal of the applied field $\mathbf{B}_{\mathrm{ip}}$, confirming its magnetic origin. All these observations are in agreement with the detected $\mathrm{THz}$ traces generated as a consequence of spin-diffusion from $\mathrm{GdFeCo}$ to Pt. Note that the laser induced demagnetization dynamics can also give rise to the emission of $\mathrm{THz}$ radiation depending on the magnetization direction, ${ }^{13,14}$ which is fixed by the externally applied magnetic field. As a consequence, the THz electric field resulting from demagnetization dynamics should not change sign with the $180^{\circ}$ reversal of the sample around its vertical axis and we can therefore exclude it as the dominant source of $\mathrm{THz}$ emission for our sample.
Next in Fig. 2(a), we show the temperature dependence of the peak electric field with an in-plane magnetic field of $\pm 0.5 \mathrm{~T}$. It is seen that around $200 \mathrm{~K}$, the sign of the electric field reverses showing that the spin-polarization injected into $\mathrm{Pt}$ also reverses its projection on the applied magnetic field. This unambiguously shows that it is not the net magnetization that determines the polarization of the spin-photocurrent from GdFeCo to Pt. For completeness, we included in Fig. 2(b) the temperature dependence of the amplitude and phase of the $\mathrm{THz}$ electric field odd in magnetic field, integrated between 0.8 and $1.2 \mathrm{THz}$. We can see that around $200 \mathrm{~K}$, there is a sharp change in the phase, indicating the sign reversal of the electric field.
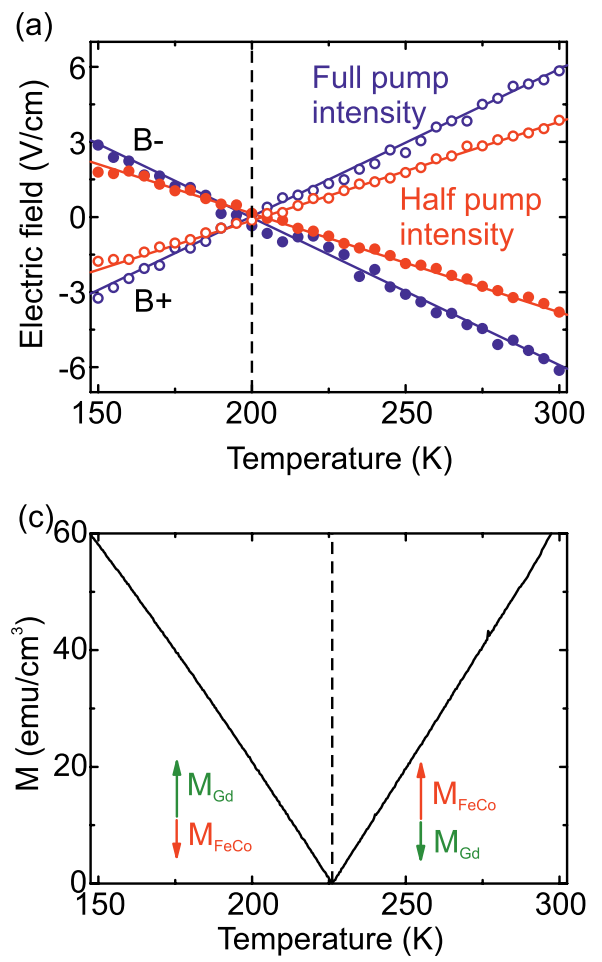

(b)

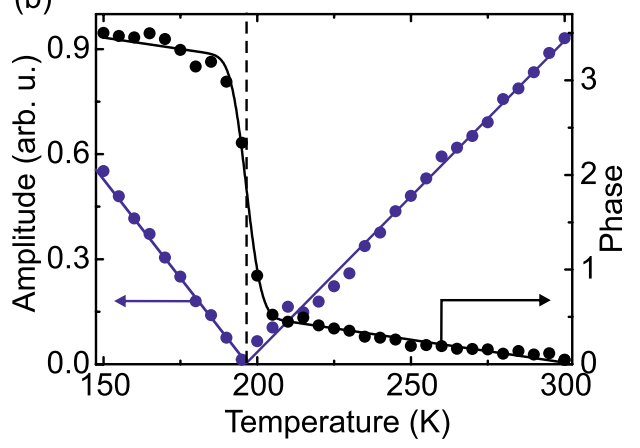

(d)

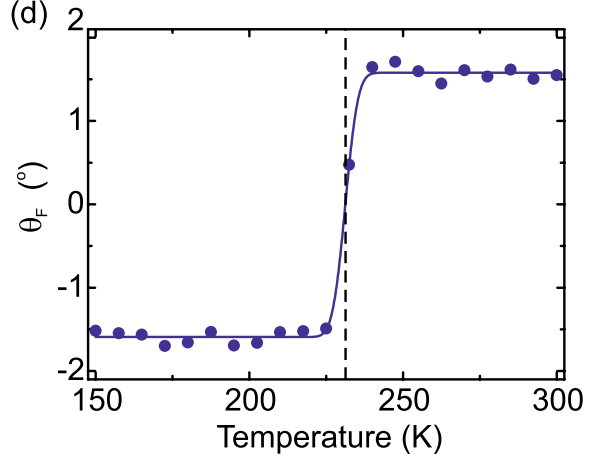

FIG. 2. (a) Temperature dependence of the emitted electric field of the $\mathrm{GdFeCo} / \mathrm{Pt}$ sample, as a function of temperature. The closed symbols correspond to an applied field of $-0.5 \mathrm{~T}$, while the open symbols correspond to an applied field of $0.5 \mathrm{~T}$. For the blue data set, the fluence of the pump was roughly twice (about $3 \mathrm{~mJ} / \mathrm{cm}^{2}$ ) the power used for the red data. The linear regression overlaying the data sets is used to guide the eye. (b) Amplitude and phase of the emitted electric field shown in (a), odd in applied magnetic field. The spectra are integrated between 0.8 and $1.2 \mathrm{THz}$. The solid lines overlaying the data sets are used to guide the eye. (c) The magnetization as measured with a vibrating sample magnetometer. (d) The temperature dependence of the Faraday rotation, measured using $\mathrm{THz}$ radiation and integrated between 0.5 and $0.9 \mathrm{THz}$. The solid lines overlaying the data set are used to guide the eye. 
Another interesting observation is that the electric field of the THz radiation crosses zero around $200 \mathrm{~K}$, which is around $25 \mathrm{~K}$ below the magnetization compensation point as measured by a vibrating sample magnetometer (VSM) shown in Fig. 2(c). In order to exclude any effect induced by cumulative heating from the laser, we have repeated the measurement for a pump power reduced by a half as shown in Fig. 2(a), which showed that the zero crossing of the $\mathrm{THz}$ emission is nearly pump intensity independent. However, Fig. 2(d) shows that the measured magneto-optical Faraday effect in the THz spectral range for this sample is characterized by an abrupt change near $\mathrm{T}_{\text {comp }}$ as measured with the VSM, similar to what we show in Ref. 15 . Since both the $\mathrm{THz}$ emission and $\mathrm{THz}$ Faraday rotation are measured in the same setup, we can exclude the influence of errors in the temperature determination. This indicates that the apparent shift of $\mathrm{T}_{\text {comp }}$ is an inherent effect of the $\mathrm{THz}$ generation mechanism.

One can write the $\mathrm{THz}$ amplitude as being proportional to a linear combination of the in-plane components of the $\mathrm{Gd}$ and FeCo magnetic sublattices, $a \mathbf{M}_{\mathrm{Gd}}+b \mathbf{M}_{\mathrm{Fe}}$. In this case, one expects zero $\mathrm{THz}$ emission when $a \mathbf{M}_{\mathrm{Gd}}+b \mathbf{M}_{\mathrm{Fe}}=0$, which does not necessarily correspond to the case in which $\mathbf{M}_{\mathrm{Gd}}+\mathbf{M}_{\mathrm{Fe}}=0$, because the sign or value of $a$ and $b$ may differ. By assuming that the magnetic anisotropy of both magnetic sublattices near the magnetization compensation temperature is similar, the fact that we see a sign change in the $\mathrm{THz}$ emission below the compensation temperature indicates that $|a|<|b|$. This means that the FeCo magnetic sublattice gives a larger contribution to the $\mathrm{THz}$ emission. This can be understood because the $\mathrm{THz}$ emission comes from a laser induced spin-polarized current and the magnetization of the $\mathrm{FeCo}$ magnetic sublattice comes from the itinerant $3 d$ electrons, while the magnetization of the Gd magnetic sublattices comes from the buried $4 f$ electrons.

Fig. 3 shows the peak THz emission for different magnetic fields and two indicative temperatures. The magnetic field dependence of the $\mathrm{THz}$ peak is reversed in sign as we cross the compensation point. Also, we note that the peak $\mathrm{THz}$ emission tends to decrease with an applied field stronger than $2 \mathrm{~T}$. While the magnetization always tends to increase with increasing magnetic field, the amount of rotation from

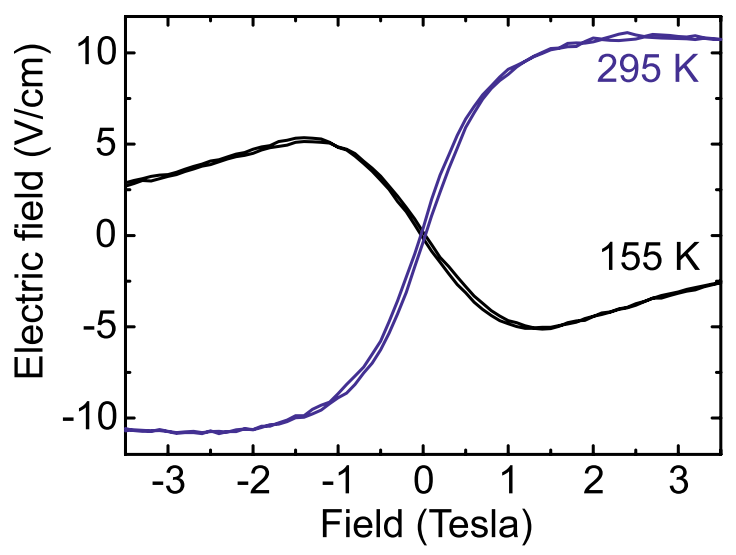

FIG. 3. Field dependencies of the GdFeCo/Pt sample. The measured electric field of the sample is observed as a function of applied field for two temperatures. out-of-plane to in-plane of the magnetic sublattices may change at finite applied magnetic fields. For small applied fields, the magnetic sublattices will typically start to align with the orientation of the applied magnetic field, while remaining more or less anti-parallel with respect to each other. However, for higher applied magnetic fields, a significant canting between the magnetic sublattices may be induced with ultimately the magnetic sublattices aligning parallel to each other and the applied magnetic field. The non-linear rotation of the magnetic sublattices as a function of field combined with magnetic sublattice sensitivity for the THz emission explains why for higher fields the amplitude of the $\mathrm{THz}$ electric field can decrease or even reverse sign.

In summary, we have shown $\mathrm{THz}$ emission arising from laser induced spin-diffusion from ferrimagnetic GdFeCo to nonmagnetic $\mathrm{Pt}$, followed by electric current generation inside the Pt capping layer by means of the inverse spin-Hall effect. Using temperature and field dependent measurements, we have shown that this emission does not follow the net magnetization but rather is dominated by a single, more itinerant, magnetic sublattice. We foresee that this magnetic sublattice sensitivity could be used as a way to generate a current ultrafast via the inverse spin-Hall effect in fully compensated metallic antiferromagnets. One may even obtain control over the magnetic sublattice sensitivity by tuning the wavelength of an optical excitation resonant to the different elements making up for the different magnetic sublattices. Moreover, particularly $\mathrm{GdFeCo}$ is an interesting material for ultrafast spintronics, as ultrafast all-optical magnetization reversal has been demonstrated with this material. ${ }^{16-20}$ Our demonstration of an ultrafast current with this material capped with Pt and in combination with all-optical magnetization reversal allows for new concepts to realize ultrafast spintronics.

We would like to thank T. Toonen and S. Semin for technical support. This work was supported by the Foundation for Fundamental Research on Matter (FOM), the European Unions Seventh Framework Program (FP7/20072013) Grant No. 280555 (Go-Fast) and No. 281043 (FemtoSpin), European Research Council Grant No. 257280 (Femtomagnetism) and Grant No. 339813 (Exchange), the program "Leading Scientist" of the Russian Ministry of Education and Science (14.Z50.31.0034), and a Leverhulme Trust (IN-2015-022).

\footnotetext{
${ }^{1}$ A. Fert, "Nobel Lecture: Origin, development, and future of spintronics," Rev. Mod. Phys. 80, 1517 (2008).

${ }^{2}$ T. Jungwirth, X. Marti, P. Wadley, and J. Wunderlich, "Antiferromagnetic spintronics," Nat. Nanotechnol. 11, 231 (2016).

${ }^{3}$ T. Kampfrath, M. Battiato, P. Maldonado, G. Eilers, J. Nötzold, S. Mährlein, V. Zbarsky, F. Freimuth, Y. Mokrousov, S. Blügel, M. Wolf, I. Radu, P. M. Oppeneer, and M. Münzenberg, "Terahertz spin current pulses controlled by magnetic heterostructures," Nat. Nanotechnol. 8, 256 (2013).

${ }^{4}$ T. J. Huisman, R. V. Mikhaylovskiy, J. D. Costa, F. Freimuth, E. Pa, J. Ventura, P. P. Freitas, S. Blügel, Y. Mokrousov, Th. Rasing, and A. V. Kimel, "Femtosecond control of electric currents in metallic ferromagnetic heterostructures," Nat. Nanotechnol. 11, 455 (2016).

${ }^{5}$ T. Seifert, S. Jaiswal, U. Martens, J. Hannegan, L. Braun, P. Maldonado, F. Freimuth, A. Kronenberg, J. Henrizi, I. Radu, E. Beaurepaire, Y. Mokrousov, P. M. Oppeneer, M. Jourdan, G. Jakob, D. Turchinovich, L. M. Hayden, M. Wolf, M. Münzenberg, M. Kläui, and T. Kampfrath,
} 
"Efficient metallic spintronic emitters of ultrabroadband terahertz radiation," Nat. Photonics 10, 483 (2016).

${ }^{6}$ D. Yang, J. Liang, C. Zhou, L. Sun, R. Zheng, S. Luo, Y. Wu, and J. Qui, "Powerful and tunable $\mathrm{THz}$ emitters based on the $\mathrm{Fe} / \mathrm{Pt}$ magnetic heterostructure," Adv. Opt. Mater. 4, 1944-1949 (2016).

${ }^{7}$ M. Battiato, K. Carva, and P. M. Oppeneer, "Superdiffusive spin transport as a mechanism of ultrafast demagnetization," Phys. Rev. Lett. 105, 027203 (2010).

${ }^{8}$ G. Malinowski, F. Dalla Longa, J. H. H. Rietjens, P. V. Paluskar, R. Huijink, H. J. M. Swagten, and B. Koopmans, "Control of speed and efficiency of ultrafast demagnetization by direct transfer of spin angular momentum," Nat. Phys. 4, 855 (2008).

${ }^{9}$ A. Melnikov, I. Razdolski, T. O. Wehling, E. Th. Papaioannou, V. Roddatis, P. Fumagalli, O. Aktsipetrov, A. I. Lichtenstein, and U. Bovensiepen, "Ultrafast transport of laser-excited spin-polarized carriers in Au/Fe/Mgo(001)," Phys. Rev. Lett. 107, 076601 (2011).

${ }^{10}$ D. Rudolf, C. La-O-Vorakiat, M. Battiato, R. Adam, J. M. Shaw, E. Turgut, P. Maldonado, S. Mathias, P. Grychtol, H. T. Nembach, T. J. Silva, M. Aeschlimann, H. C. Kapteyn, M. M. Murnane, C. M. Schneider, and P. M. Oppeneer, "Ultrafast magnetization enhancement in metallic multilayers driven by superdiffusive spin current," Nat. Commun. 3, 1037 (2012).

${ }^{11}$ G.-M. Choi, B.-C. Min, K.-J. Lee, and D. G. Cahill, "Spin current generated by thermally driven ultrafast demagnetization," Nat. Commun. 5, 4334 (2014).

${ }^{12} \mathrm{G}$. Gallot and Grischkowsky, "Electro-optic detection of terahertz radiation," J. Opt. Soc. Am. B 16, 1204 (1999).

${ }^{13}$ E. Beaurepaire, G. M. Turner, S. M. Harrel, M. C. Beard, J.-Y. Bigot, and C. A. Schmuttenmaer, "Coherent terahertz emission from ferromagnetic films excited by femtosecond laser pulses,” Appl. Phys. Lett. 84, 3465 (2004).
${ }^{14}$ T. J. Huisman, R. V. Mikhaylovskiy, A. Tsukamoto, T. Rasing, and A. V. Kimel, "Simultaneous measurements of terahertz emission and magnetooptical Kerr effect for resolving ultrafast laser-induced demagnetization dynamics," Phys. Rev. B 92, 104419 (2015).

${ }^{15}$ T. J. Huisman, R. V. Mikhaylovskiy, A. Tsukamoto, L. Ma, W. J. Fan, S. M. Zhou, Th. Rasing, and A. V. Kimel, "Sub 100-ps dynamics of the anomalous Hall effect at THz frequencies," e-print arXiv:1611.06251.

${ }^{16}$ C. D. Stanciu, F. Hansteen, A. V. Kimel, A. Kirilyuk, A. Tsukamoto, A. Itoh, and T. Rasing, "All-optical magnetic recording with circularly polarized light,” Phys. Rev. Lett. 99, 047601 (2007).

${ }^{17}$ K. Vahaplar, A. M. Kalashnikova, A. V. Kimel, D. Hinzke, U. Nowak, R. Chantrell, A. Tsukamoto, A. Itho, A. Kirilyuk, and T. Rasing, "Ultrafast path for optical magnetization reversal via a strongly nonequilibrium state," Phys. Rev. Lett. 103, 117201 (2009).

${ }^{18}$ I. Radu, K. Vahaplar, C. Stamm, T. Kachel, N. Pontius, H. A. Dürr, T. A. Ostler, J. Barker, R. F. L. Evans, R. W. Chantrell, A. Tsukamoto, A. Itoh, A. Kirilyuk, Th. Rasing, and A. V. Kimel, "Transient ferromagnetic-like state mediating ultrafast reversal of antiferromagnetically coupled spins," Nature 472, 205 (2011).

${ }^{19}$ M. Savoini, R. Medapalli, B. Koene, A. R. Khorsand, L. Le Guyader, L. Duò, M. Finazzi, A. Tsukamoto, A. Itoh, F. Nolting, A. Kirilyuk, A. V. Kimel, and Th. Rasing, "Highly efficient all-optical switching of magnetization in GdFeCo microstructures by interference-enhanced absorption of light," Phys. Rev. B 86, 140404 (2012).

${ }^{20}$ C. E. Graves, A. H. Reid, T. Wang, B. Wu, S. de Jong, K. Vahaplar, I. Radu, D. P. Bernstein, M. Messerschmidt, L. Müller et al., "Nanoscale spin reversal by non-local angular momentum transfer following ultrafast laser excitation in ferrimagnetic GdFeCo," Nat. Mater. 12, 293 (2013). 